\title{
First-principles investigation of the stability of $3 d$ monolayer/Fe(001) against bilayer formation
}

\author{
T. Asada ${ }^{a}$ \\ Faculty of Engineering, Shizuoka University, Hamamatsu 432-8561, Japan \\ S. Blügel, G. Bihlmayer, S. Handschuh, and R. Abt \\ Institut für Festkörperforschung, Forschungszentrum Jülich, D-52425 Jülich, Germany
}

\begin{abstract}
Based on the full-potential linearized augmented plane-wave method combined with the generalized gradient approximation, we determine the ground-state spin configurations and the total energies of $3 d$ transition- metal monolayer and bilayer films on $\mathrm{Fe}(001)$ within the $c(2 \times 2)$ unit cell. We find by energy analysis that $\mathrm{V}, \mathrm{Cr}$, and $\mathrm{Mn}$ layers prefer the layered antiferromagnetic coupling, and $\mathrm{Fe}$, $\mathrm{Co}$, and $\mathrm{Ni}$ layers favor the ferromagnetic coupling to $\mathrm{Fe}(001)$. One exception is the $\mathrm{Mn}$ monolayer, which favors the $c(2 \times 2)$ ferrimagnetic superstructure. We discuss the stability of the $3 d$ transition-metal monolayer films on $\mathrm{Fe}(001)$ against the bilayer formation and find that, with the exception of $\mathrm{Cr}$, all $3 d$ monolayers on $\mathrm{Fe}(001)$ are stable against bilayer formation. We have confirmed that the interlayer relaxations do not change the overall features of the present results. (C) 2000 American Institute of Physics. [S0021-8979(00)48708-6]
\end{abstract}

\section{INTRODUCTION}

$3 d$ metal monolayers (ML) on $\mathrm{Fe}(001)$ are prototypical systems where in-plane magnetic interactions compete with strong magnetic interactions between the ML and the Fe substrate. In particular the magnetism of ultrathin films of $\mathrm{Cr}$ and $\mathrm{Mn}$ on $\mathrm{Fe}(001)$ are under intense scrutiny for the understanding of the interfacial magnetism. The experimental results are still partly in contradiction and the complexity of those systems have been gradually revealed. For a Cr ML the measured magnetic moment was found to be at most 1 $\mu_{\mathrm{B}},{ }^{1,2}$ which is less than half of the theoretical prediction., ${ }^{3,4}$ It was also reported that a layer-by-layer growth leads to a strong intermixing with the substrate Fe layers. ${ }^{5-7} \mathrm{Mn}$ overlayers seem to be even much more involved. There is a general experimental consensus that for Mn around $1 \mathrm{ML}$ coverage the signals related to the magnetization disappear. The microscopic origin for this observation is under strong debates. The difficulty in controlling and characterizing the morphology of the interface seems to be intimately related to the difficulties in the understanding of the interfacial magnetism of those systems.

This article is dedicated to shed some light on the aspect of the stability of the $3 d$ metal MLs on Fe(001) with respect to the bilayer (BL) formation. So far no ab initio calculations on the $3 d$ overlayers on $\mathrm{Fe}(001)$ have focused on the stability arguments of these systems. We calculated the magnetic moments, the magnetic structures, and the total energies for two types of $3 d$ metal systems: (i) the $3 d$ MLs on Fe(001) and (ii) the $3 d$ BLs on $\mathrm{Fe}(001)$, and in addition the plain $\mathrm{Fe}(001)$ without $3 d$ overlayers. Each type of systems includes the $3 d$ metals $\mathrm{V}, \mathrm{Cr}, \mathrm{Mn}, \mathrm{Fe}, \mathrm{Co}$, and Ni. By comparing the total energy of MLs on $\mathrm{Fe}(001)$ with the one of BLs covering $50 \%$ of the Fe surface and leaving $50 \%$ uncovered, we extract the BL formation energy as a measure of the

aElectronic address: tstasad@ipc.shizuoka.ac.jp stability of the $3 d$ MLs on $\mathrm{Fe}(001)$ against BL formation during growth or after annealing. Since what occurs in real growth would be strongly governed by the energy barriers of elementary processes, we expect that the examination of such total energy aspects will give a good estimation of how systems evolve for systems where the energy barriers are sufficiently low. Therefore, we may also expect that comparisons of the present examinations with experimental growth behaviors will give some indications of such barrier heights.

\section{METHOD}

The calculations were carried out with the full-potential linearized augmented plane-wave (FLAPW) program FLEUR in film geometry ${ }^{8}$ combined with the generalized gradient approximation (GGA) proposed by Perdew et al. ${ }^{9}$ Two film geometries were considered, the first consisted of seven bcc $\mathrm{Fe}(001)$ layers and one $3 d$ metal ML on each side of the $\mathrm{Fe}(001)$ surface, and the second consisted of seven $\mathrm{Fe}(001)$ layers with two $3 d$ metal MLs on each side. We searched for the ground-state spin configurations within a $c(2 \times 2)$ unit cell excluding noncollinear magnetic structures. Prior to the ML and BL calculations we determined the theoretical (GGA-FLAPW) equilibrium lattice constant of FM bcc Fe as $a_{0}=5.33$ a.u., which is smaller by $1.5 \%$ as compared with the experimental value of 5.41 a.u. Thus, the calculations are carried out with the in-plane lattice constant of 5.33 a.u. and results of structurally unrelaxed calculations use the interlayer distance of 2.665 a.u. Converged total energies were obtained by use of roughly 70 symmetrized augmented plane waves per atom as a variational basis set and 36 and 21 special $k_{\|}$points for the $p(1 \times 1)$ and $c(2 \times 2)$ unit cells, respectively, to integrate over the irreducible wedge of the two-dimensional Brillouin zone. To determine the magnetic ground-state configurations of each system we have examined all conceivable spin configurations. The interlayer dis- 


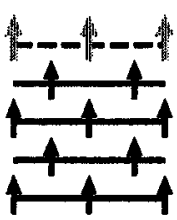

ferromagnetic

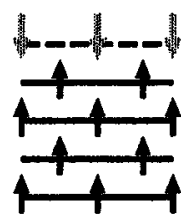

layered antiferro.

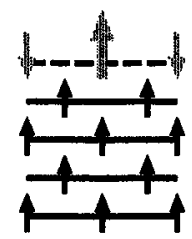

c(2x2) ferrimagn.
FIG. 1. Schematic side-view representation of a FM, a LAF, and a $c(2$ $\times 2$ ) FI superstructure of a ML film (broken line) grown as overlayer on a magnetic substrate (full line). Arrows indicate the relative spin direction at the positions of the atoms.

tances of relevant magnetic configurations of the ML and BL systems are fully optimized by use of force calculations.

Figure 1 shows the three collinear magnetic configurations conceivable for the ML systems in the $c(2 \times 2)$ unit cell: the $p(1 \times 1)$ ferromagnetic $(\mathrm{FM}),(\uparrow \mid \uparrow \mathrm{Fe})$, the $p(1$ $\times 1)$ layered antiferromagnetic (LAF), $(\downarrow \mid \uparrow \mathrm{Fe})$, and the $c(2 \times 2)$ ferrimagnetic $(\mathrm{FI})$ spin configuration, $([\uparrow \downarrow] \mid \uparrow \mathrm{Fe})$. For the $\mathrm{BL}$ systems there are eight magnetic configurations in the $c(2 \times 2)$ unit cell: the $p(1 \times 1)(\mathrm{FM}),\left(\uparrow_{s} \uparrow_{s-1} \mid \uparrow \mathrm{Fe}\right)$, the $p(1 \times 1) \mathrm{LAF},\left(\uparrow_{s} \downarrow_{S-1} \mid \uparrow \mathrm{Fe}\right)$, as well as the spinflipped $p(1 \times 1)$ configurations $\left(\downarrow_{S} \downarrow_{S-1} \mid \uparrow \mathrm{Fe}\right)$, and $\left(\downarrow_{S}\right.$ $\uparrow s-1 \mid \uparrow \mathrm{Fe})$, and four $c(2 \times 2)$ FI spin configurations, the $\left([\uparrow \downarrow]_{S} \uparrow_{S-1} \mid \uparrow \mathrm{Fe}\right)$, the $\left(\uparrow_{S}[\uparrow \downarrow]_{S-1} \mid \uparrow \mathrm{Fe}\right)$, as well as the two spin-flipped configurations $\left([\uparrow \downarrow]_{S} \downarrow_{S-1} \mid \uparrow \mathrm{Fe}\right)$, and $\left(\downarrow_{S}\right.$ $\left.[\uparrow \downarrow]_{S-1} \mid \uparrow \mathrm{Fe}\right)$.

\section{RESULTS}

We show in Fig. 2 the magnetic moments for FM, LAF, and FI spin structures of the unrelaxed $3 d$ MLs on Fe(001). The magnetic moments of the interface Fe atom for groundstate spin configurations are also shown. The magnetic ground-states are LAF for $\mathrm{V}$ and $\mathrm{Cr}$, FI for $\mathrm{Mn}$, FM for Fe, $\mathrm{Co}$, and $\mathrm{Ni}$, as has been reported in the literature. ${ }^{3,4,10}$ For Mn MLs the magnetic moment of the interface Fe atom is reduced making a distinguished dip. This is a result of $\mathrm{Mn}$ being a transient element between an AF and a FM coupling

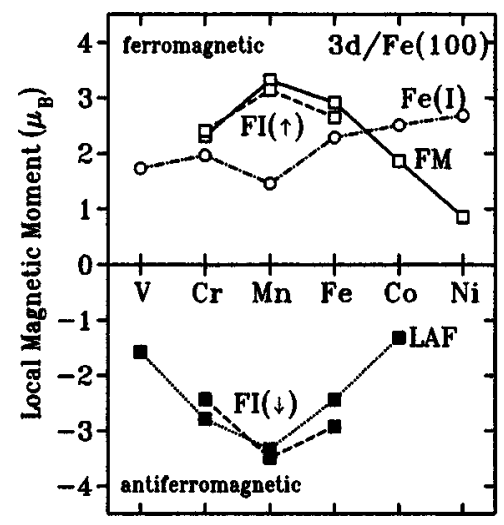

FIG. 2. Local magnetic moments of unrelaxed $3 d$ transition-metal MLs on $\mathrm{Fe}(001)$. Positive (negative) sign of moments indicates a FM(AF) spin alignment to the Fe substrate, emphasized by open (filled) symbols. Shown are three spin configurations, $p(1 \times 1)$ FM (solid line), $p(1 \times 1)$ LAF (dotted line), and the $c(2 \times 2)$ FI (dashed lines) for positive and negative moments. Included are the moments of interface Fe (chained line) for the ground-state spin configurations.

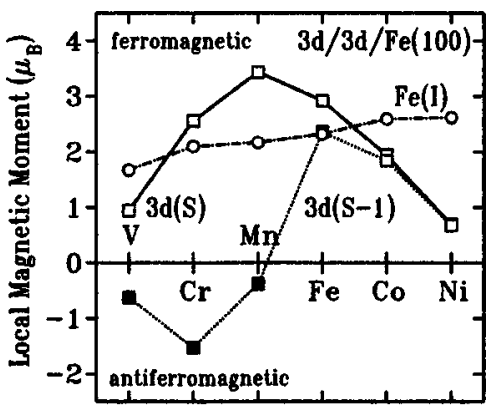

FIG. 3. Local magnetic moments $(M)$ for the ground-state spin configurations of unrelaxed $3 d$ transition-metal BLs on $\mathrm{Fe}(001)$. The solid line denotes the $3 d$ surface atoms $[3 d(\mathrm{~S})]$ aligned parallel $(M>0)$ to the Fe substrate, the dotted line denotes the $3 d$ subsurface atoms [3d(S-1)], whose coupling changes from LAF $(M<0)$ to FM $(M>0)$ from early to late $3 d$ elements. The chained line denotes the interface $\mathrm{Fe}$ atoms $[\mathrm{Fe}(\mathrm{I})]$.

between early and late $3 d$ transition-metal elements, respectively, as discussed by Mirbt et al. ${ }^{3}$ Here the size of the dip at $\mathrm{Mn}$ is a little bit larger than the one calculated by Mirbt et al. owing to the present incorporation of the $c(2 \times 2)$ configuration. The total energy differences between FM and LAF configurations and between FM and FI show some differences to those of Handschuh and Blügel ${ }^{4}$ coming mostly from the different choice of the in-plane lattice constant. Extending the search for the magnetic ground-state of $\mathrm{Mn}$ to larger surface unit-cells will most likely lead to a more complicated spin-structure ${ }^{11-13}$ not discussed here any further.

For the BL systems we have examined all possible magnetic configurations within the $c(2 \times 2)$ unit cell. With the exception of $\mathrm{Mn}$ all stable magnetic solutions found have the $p(1 \times 1)$ structure. The magnetic ground-states of the $3 d$ BLs are the $p(1 \times 1)$ LAF one, $\left(\uparrow_{\mathrm{s}} \downarrow_{s-1} \mid \uparrow \mathrm{Fe}\right)$, for $\mathrm{V}, \mathrm{Cr}$, and $\mathrm{Mn}$, and the $p(1 \times 1) \mathrm{FM}$ one for $\mathrm{Fe}, \mathrm{Co}$, and $\mathrm{Ni}$, as seen in the signs of magnetic moments shown in Fig. 3. For the case of the LAF Mn BL we observe an almost vanishing magnetic moment of the subsurface Mn atom. For Mn, in addition to the ground-state structure, we found another LAF configuration, $\left(\downarrow_{S} \uparrow_{S-1} \mid \uparrow \mathrm{Fe}\right)$, about $9 \mathrm{meV} / \mathrm{Mn}$ higher in energy than the ground state, which is the one found by $\mathrm{Wu}$ and Freeman. ${ }^{13}$ Furthermore other than these two LAF structures we found a superstructure $\left([\uparrow \downarrow]_{S} \downarrow_{S-1} \mid \uparrow \mathrm{Fe}\right)$ as a stable solution with the total energy higher than the ground state by $43.2 \mathrm{meV} / \mathrm{Mn}$. Thus, there exist three states within an energy range corresponding to about $400 \mathrm{~K}$, which seems to show already a part of difficulties involved in the calculation of thicker Mn overlayers on $\mathrm{Fe}(001) .{ }^{14}$

In Fig. 4 we show the BL formation energy defined as the total energy difference of three systems, $E_{\mathrm{ML} / \mathrm{Fe}}$ $-1 / 2\left[E_{\mathrm{BL} / \mathrm{Fe}}+E_{\mathrm{Fe}(001)}\right]$, whose negative value corresponds to the case where ML formation is preferred. The figure indicates that, with the exception of $\mathrm{Cr}$, all $3 d$ transition metals favor the ML formation over the BL formation. Though this overall trend is already visible in the nonmagnetic results, the strong magnetic effect for Mn changes the sign of its BL formation energy. The results seem to meet the experimental observation on the $\mathrm{Cr}$ overlayer where its ML is unstable. ${ }^{6}$ For other $3 d$ overlayers ML stability assessment requires 


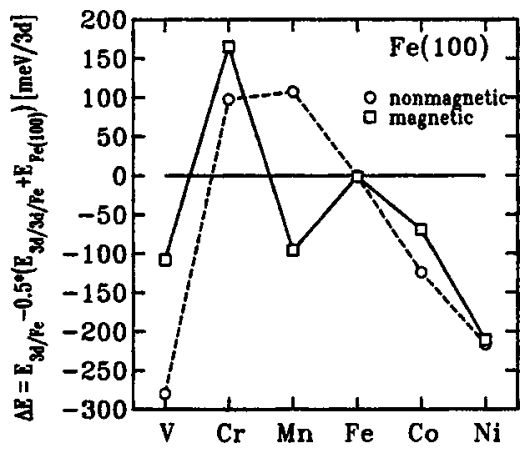

FIG. 4. Shown is the bilayer formation energy $\Delta E$ per $3 d$ transition-metal atom for unrelaxed geometries. For $\Delta E<0(>0)$, ML (BL) is energetically preferred. The solid (dashed) line denotes results derived from the (non)magnetic ground-state spin configuration within the $c(2 \times 2)$ unit cell. The interlayer relaxations do not change the overall features of this figure.

extension of the present investigations to, e.g., the effect of interdiffusion, which are now in progress.

\section{CONCLUSION}

In summary, we have determined by $a b$ initio total energy and force calculations the ground-state spin configurations and the total energies of $3 d$ transition-metal ML and BL films on $\mathrm{Fe}(001)$. We found that for both MLs and BLs $\mathrm{V}, \mathrm{Cr}$, and $\mathrm{Mn}$ prefer the $p(1 \times 1) \mathrm{LAF}$ configuration and $\mathrm{Fe}$, $\mathrm{Co}$, and $\mathrm{Ni}$ the $p(1 \times 1)$ FM with a single exception of the Mn ML which prefers a FI structure within the restricted choice of a $c(2 \times 2)$ unit cell. We found that all $3 d$ elements favor the ML over the BL except for Cr. The present result of the Cr ML instability against BL formation may meet the experimentally found instability of the Cr ML.

\section{ACKNOWLEDGMENTS}

This work was supported by the DFG under Grant No. BL444/1-1 and by the Program "Verbundforschung Synchrotronstrahlung" of the BMBF, and the TMR Networks, Contract Nos.: EMRX-CT96-0089 and FMRX-CT98-0178.

${ }^{1}$ F. U. Hillebrecht, Ch. Roth, R. Jungblut, E. Kisker, and A. Bringer, Europhys. Lett. 19, 711 (1992).

${ }^{2}$ R. Jungblut, Ch. Roth, F. U. Hillebrecht, and E. Kisker, J. Appl. Phys. 70, 5923 (1991).

${ }^{3}$ S. Mirbt, O. Eriksson, B. Johansson, and H. L. Skriver, Phys. Rev. B 52, 15070 (1995).

${ }^{4}$ S. Handschuh and S. Blügel, Solid State Commun. 105, 633 (1998).

${ }^{5}$ D. Venus and B. Heinrich, Phys. Rev. B 53, R1733 (1996).

${ }^{6}$ R. Pfandzelter, T. Igel, and H. Winter, Phys. Rev. B 54, 4496 (1996).

${ }^{7}$ A. Davies, J. A. Stroscio, D. T. Pierce, and R. J. Celotta, Phys. Rev. Lett. 76, 4175 (1996).

${ }^{8}$ E. Wimmer, H. Krakauer, M. Weinert, and A. J. Freeman, Phys. Rev. B 24, 864 (1981); M. Weinert, E. Wimmer, and A. J. Freeman, ibid. 26, 4571 (1982).

${ }^{9}$ J. P. Perdew, J. A. Chevary, S. H. Vosko, K. A. Jackson, M. R. Pederson, D. J. Singh, and C. Fiolhais, Phys. Rev. B 46, 6671 (1992).

${ }^{10}$ R. Wu and A. J. Freeman, Phys. Rev. B 51, 17131 (1995).

${ }^{11}$ O. Elmouhssine, G. Moraitis, C. Demangeat, and J. C. Parlebas, Phys. Rev. B 55, R7410 (1997).

${ }^{12}$ T. Asada, G. Bihlmayer, and S. Blügel (unpublished).

${ }^{13}$ R. Wu and A. J. Freeman, J. Magn. Magn. Mater. 161, 89 (1996).

${ }^{14} \mathrm{~T}$. Asada, S. Bügel, and G. Bihlmayer (unpublished). 